\title{
In vitro mutagenesis using bio-beam irradiation on in vitro culture of Cavendish banana cultivar (Musa acuminata Colla) explants
}

\begin{abstract}
Banana, Musa acuminata cv. Cavendish, was commonly propagated by use of suckers from selected field grown clumps. An in vitro technique has in recent years provided with rapid and efficient system propagation. However, high demand for planting material has caused significant shortages and subsequently attempts at improving the efficiency of in vitro propagation technique have been initiated. The current study investigated the effect of gamma irradiation using bio-beam on in vitro propagation of banana cv. Cavendish. The main objective was to assess the effect of mutation induction using gamma source on growth and development of plantlets while in culture. Tissue cultured banana plantlets were used as source of explant materials. The plantlet was trimmed of leaves and halved before culturing on Murashige and Skoog (MS) media. Then, the explants radiated with gamma source using Ion-Beam with selected dose [0 (control), 30 Gy, 60 Gy, 90 Gy, 120 Gy and $150 \mathrm{~Gy}$ ] and transferred to fresh MS media with $5 \mathrm{mg} / \mathrm{l}$ of BAP. The analysis showed dosage of $30 \mathrm{~Gy}$ and $60 \mathrm{~Gy}$ produced significant survival rate and favourable effect on growth especially on plant height, shoot number, leaf production and root production. Thus, this study contributed to develop in vitro mutagenesis of M. acuminata cv. Cavendish through gamma rays coupled with in vitro system for future breeding of new varieties of banana.
\end{abstract}

Keyword: Bio-beam; Gamma irradiation; In vitro propagation; LD5; Musa acuminata cv. cavendish; Mutation 